\title{
Anabases
}

ANABASES Traditions et réceptions de l'Antiquité

5 | 2007

Varia

\section{Jérôme Carcopino, un historien dans Rome}

\section{Sarah Rey}

\section{OpenEdition}

Journals

Édition électronique

URL : http://journals.openedition.org/anabases/3194

DOI : 10.4000/anabases.3194

ISSN : 2256-9421

\section{Éditeur}

E.R.A.S.M.E.

\section{Édition imprimée}

Date de publication : 1 mars 2007

Pagination : 191-206

ISSN : 1774-4296

\section{Référence électronique}

Sarah Rey, « Jérôme Carcopino, un historien dans Rome », Anabases [En ligne], 5 | 2007, mis en ligne le 01 octobre 2012, consulté le 21 octobre 2019. URL : http://journals.openedition.org/anabases/3194 ; DOI : 10.4000/anabases.3194 


\section{Jérôme Carcopino, un historien dans Rome}

SARAH REY

Comme de nombreux spécialistes de la Rome antique, Jérôme Carcopino a accompli une forme de "voyage d'Italie ». Membre de l'École française de Rome (19041907), directeur intérimaire de cette institution (1922-1923) avant d'en être le directeur à part entière (1937-1940), il est notamment l'auteur d'un Sylla ou la monarchie manquée ${ }^{1}$, d'un César ${ }^{2}$, et des Secrets de la correspondance de Cicéron ${ }^{3}$. Il a voulu relater ses années à l'École française dans ses Souvenirs romains ${ }^{4}$. Plus qu'un voyage, Carcopino a effectué en Italie une série de séjours d'initiation et de perfectionnement : il est venu se mettre au contact des antiquités italiennes. À la différence des écrivains, des fonctionnaires expatriés, des touristes, l'Italie n’a pu, pour lui, être temporaire. Dans son cas, Rome n’a pas seulement constitué un passage dans sa carrière, Rome n’a pas été qu'une simple curiosité.

Jérôme Carcopino est dans la Ville en tant qu'historien. Rome est présente du début à la fin de sa vie : depuis l'agrégation jusqu'à ses derniers moments. En effet, les deux derniers visiteurs qu'il reçoit à quelques heures de sa mort sont Pierre Grimal, son élève à Rome, et André François-Poncet ${ }^{5}$, qui occupe le poste d'ambassadeur de France en Italie quand Carcopino est à la direction du Farnèse.

1 J. CarCopino, Sylla ou la monarchie manquée, Paris, L'artisan du livre, 1931, 248 p.

2 Le premier César est dans G. Glotz (dir.), Histoire ancienne, $3^{e}$ partie. Histoire romaine. Id., Les Secrets de la correspondance de Cicéron, Paris, L'artisan du livre, 1947, 2 vol., 446 p. et $494 \mathrm{p}$.

$4 \quad$ Id., Souvenirs romains, Paris, Hachette, 1968, 292 p.

5 C'est à André François-Poncet, "à l'Ambassadeur et au Normalien ", que Jérôme Carcopino dédie Passion et politique chez les Césars (Paris, Hachette, 1958, 222 p.). 
Continûment, Rome prend place dans son parcours, mais quelle Rome ? Rome représente d'abord un choix datant de l'École normale, lorsque l'apprenti historien est repéré pour son goût du latin. Rome est un terrain de recherches et sert, en outre, de tremplin dans la course aux honneurs que Carcopino poursuit sans cesse. Mais son intérêt pour l'histoire romaine l'encourage-t-il à étudier la ville de Rome en elle-même ? Et comment cerne-t-il l'Urbs ? Un ouvrage, écrit au moment de sa direction de l'EFR, la Vie quotidienne à Rome à l'apogée de l'Empire (1939) 6, permet d'avancer ici des éléments de réponse.

\section{Le choix de Rome}

Jérôme Carcopino aurait pu être spécialiste de la Grèce, mais il a préféré s'orienter vers l'antiquité romaine pour des raisons exposées dans les Souvenirs romains. Dans ce livre autobiographique, Carcopino se remémore les circonstances qui ont présidé à ses débuts dans le métier d'historien : entrent alors en ligne de compte des questions de compétence philologique, ajoutées à un prétexte des plus minces (le dessin). Par-dessus tout, la conviction d'une supériorité de l'histoire de Rome joue. Pour compléter la mise en scène de ce moment important, le romaniste fait parler Georges Perrot, historien de l'art grec, et directeur de la rue d'Ulm quand Carcopino est élève normalien :

Vous avez utilisé [dit Perrot, rapporté par Carcopino] les heures de sortie que vous concédait le règlement [de l'École normale] pour suivre au Collège de France les cours d'épigraphie latine de Cagnat. Eh bien! soyez tranquille! Avec l'accord de Mgr Duchesne, votre futur directeur du Palais Farnèse, je vais soumettre votre nom au Ministre pour la place dont je dispose dans la promotion romaine, mais je le regrette. Car, enfin, vous êtes intelligent, Carcopino ; et Bouché-Leclercq a partout vanté votre mémoire de licence sur l'ostracisme. Je n'arrive pas à comprendre que vous ayez préféré Rome à Athènes. Enfin puisque vous le voulez... Il n'eut pas le courage d'achever sa phrase [...] Je m'abstins d'invoquer l'attrait que, comparée aux luttes locales dont sont remplies les annales grecques, l'étonnante expansion de la puissance romaine en un empire universel avait exercé sur ma vocation. Je n'eus pas à exagérer ma maladresse de dessinateur qui m’avait détourné de l'Histoire de l'Art ; et je fis état de ma faiblesse philologique en grec alors que mes maîtres m'estimaient excellent latiniste. Finalement, j'avais mieux aimé être un bon ouvrier de l'histoire romaine que trahir l'histoire grecque par mes insuffisances 7 .

De cet extrait il ressort que le jeune Carcopino subit l'influence des conceptions politiques et historiographiques hostiles aux cités démocratiques, préférant s'intéresser

6 J. Carcopino, La Vie quotidienne à Rome à l'apogée de l'Empire, Paris, Hachette, 1939, $342 \mathrm{p}$.

7 CARCOPINO, Souvenirs romains, p. 22. 
à une histoire politique considérée comme plus « grande » et plus digne d'être étudiée, celle de la construction du pouvoir romain.

Cette orientation prise, Carcopino part étudier Ostie, en tant que membre (19041907) de l'École française de Rome, alors sous la direction de Mgr Duchesne. Ostie forme la matière de sa thèse de doctorat d'État. Les monographies de villes antiques, comme celle que Carcopino consacre à Ostie, sont déjà et restent longtemps des sujets classiques de thèses estampillées "École française " : avant lui, Terracine, Carthage romaine, Bologne ont été étudiées ${ }^{8}$. Après lui, Capoue, et - hors d'Italie - Paris sont, par exemple, explorées ${ }^{9}$. Selon la thèse que soutient Carcopino en 1919 sur Virgile et les origines d'Ostie ${ }^{10}$, les six derniers livres de l'Enéide s'inspirent sans le dire du cadre physique d'Ostie.

Carcopino est élève de Rome en même temps qu’Albert Grenier, Eugène Albertini, Jacques Zeiller, Pierre Boudreaux, André Piganiol et François Préchac. Dans ces promotions 1904-1907, les sujets de philologie sont aussi nombreux que les sujets d'histoire : quand Grenier travaille sur Bologne, Zeiller sur les provinces danubiennes ${ }^{11}$, Piganiol sur les origines de Rome ${ }^{12}$, Albertini 13 et Préchac 14 se consacrent à Sénèque, Boudreaux à Aristophane ${ }^{15}$. En philologue, Carcopino - lui qui se veut aussi et surtout historien - fait une grande relecture de Virgile. Pour sa thèse, Jérôme Carcopino effectue une balade archéologique à la manière distinguée de Gaston Boissier ${ }^{16}$. Virgile lui vient à chaque pas. Comme d'autres membres de l'École française, il achoppe sur les autorisations limitées de fouilles données par les autorités italiennes ${ }^{17}$. Il doit se contenter de quelques prospections. Il cherche aussi à donner à l'histoire romaine une autonomie. En effet, le séjour à Rome a d'abord été conçu, pour quelques savants français,

8 R. Moulin Ducoudray De la Blanchère, Terracine. Essai d'histoire locale, Paris, De Boccard, 1884, 218 p. ; A. Audollent, Carthage romaine. 146 av. J.-C .-698 ap. J.-C., Paris, De Boccard, 1901, 850 p. ; A. GRENIER, Bologne villanovienne et étrusque, Paris, De Boccard, 1912, 540 p. D. ANZIANI, mort au combat en 1914, avait entamé, pour sa part, une thèse sur Véies.

J. Heurgon, Recherches sur l'histoire, la religion et la civilisation de Capoue préromaine, des origines à la deuxième guerre punique, Paris, De Boccard, 1942, 483 p. Voir aussi P.-M. Duval, Paris antique, des origines au III siècle, Paris, Hermann, 1961, 369 p.

J. Carcopino, Virgile et les origines d'Ostie, Paris, De Boccard, 1919, 819 p.

11 J. ZEILLER, Les origines chrétiennes dans les provinces danubiennes de l'Empire romain (Norique, les deux Pannonies et les deux Mésies), Paris, de Boccard, 1918, 667 p.

12 A. Piganiol, Essai sur les origines de Rome, Paris, De Boccard, 1916, 343 p.

13 E. Albertini, La composition dans les ouvrages de Sénèque, Paris, De Boccard, 1923, 354 p.

14 F. PreChaC, Le traité De Clementia : sa composition et sa destination, Paris, Les Belles Lettres, 1921, 48 p.

15 P. BOUDREAUX, Le texte d'Aristophane et ses commentateurs, Paris, De Boccard, 1919, 207 p.

16 Cf. par exemple G. BoIssier, Promenades archéologiques. Rome et Pompéi, Paris, 1880, 383 p.

17 Voir P. Grimal, P. Ourlac, C. CarCopino, Jérôme Carcopino, un historien au service de l'humanisme, Paris, Les Belles Lettres, 1981, p. 138 et p. 164. 
comme un préalable à l'École d'Athènes ${ }^{18}$. Avec son Virgile, Carcopino écrit comme un pendant aux Phéniciens et l'Odyssée de Victor Bérard ${ }^{19}$, avec l'idée que ce qui est fait en histoire grecque doit pouvoir trouver un parallèle en histoire romaine. Ainsi, à Ostie, il voit des biches ; "or c'est un cerf blessé qui, dans l'Énéide, sert de prétexte aux Latins pour déclarer la guerre $20 \%$. Il suit le Tibre, sent le soufre à Zolforata et en déduit que " dans ce paysage tout imprégné de crainte et de mystère, les Latins ont dû se sentir plus proches de la divinité tellurique [Vulcanus] ${ }^{21}$ ». Il retrouve, " perdus dans les roseaux de l'étang », « les débris supérieurs 22 » des colonnes de la capitainerie du port d'Ostie.

Parmi ses successeurs farnésiens, d'autres cherchent de même, et parfois de manière forcée, à retrouver l'antique ${ }^{23}$. En tout cas, l'École de Rome n'a jamais institué de visites collectives et systématiques de sites antiques, au contraire de ce qu'a pu faire l'École biblique de Jérusalem ${ }^{24}$. L'historien poursuit ce type de sorties sur le terrain tout au long de sa carrière, en Afrique du Nord et en Italie. Il découvre l'inscription d'Aïn-el-Djemala dans un oued, en Tunisie ${ }^{25}$, inscription qui aide à mieux comprendre la politique agraire d'Hadrien. Plus tard, il s'intéresse entre autres à Timgad ${ }^{26}$, ̀̀ Tipasa ${ }^{27}$, à Carthage ${ }^{28}$. Il cherche une hypothétique Ilva près de Rome,

18 Du temps de l'Institut de correspondance archéologique (fondé en 1829 et devenu totalement allemand après la guerre de 1870), les membres de l'École française d'Athènes (instituée en 1846) s'arrêtent un an à Rome avant de poursuivre leur trajet vers la Grèce. En 1873, un décret signé par Adolphe Thiers crée une "section romaine de l'École d'Athènes ". Le décret du 20 novembre 1875 établit que l'École de Rome sera constituée des futurs membres de l'École d'Athènes et de six membres strictement " romains ". Cf. par exemple C. Pietri, Ph. Boutry, F.-Ch. Uginet, "La scuola francese di Roma ”, in Speculum mundi. Roma, centro internazionale di ricerche umanistiche, Rome, Unione internazionale degli Istituti di Archeologia, 1993, p. 216. V. Berard, Les Phéniciens et l'Odyssée, Paris, Armand Colin, 2 vol., 1902-1903, 391 p. et 255 p. Prélude aux Navigations d'Ulysse, Paris, Armand Colin, 1927-1929, 591 p. et 451 p. Carcopino, Virgile et les origines d'Ostie, p. 317.

Ibid., p. 342.

J. Carcopino, Ostie. Les Visites d'art, Paris, Henri Laurens, 1929, p. 19.

Cf. P. WuilLEmier qui déclare qu’à Tarente les " chèvres qu'a rencontrées Virgile, parcourent encore aujourd'hui les rues de la ville " (Tarente, des origines à la conquête romaine, Paris, De Boccard, 1939, p. 217). Ou J. HEURGON qui, sur un même mode, compare les physionomies étrusques et toscanes, d'après les vases de Volterra (III siècle) (in La Vie quotidienne chez les Étrusques, Paris, Hachette, 1961, p. 40-41). Ponctuellement, et précisément sous la direction Carcopino, l'École a organisé pour les membres des visites de Véies et des Castelli romani. Cf. Mélanges de l'École française de Rome, 26 (1906), p. 365-481.

Voir par exemple J. CARCOPINO, " La table de patronat de Timgad ", Revue africaine, 57 (1913), p. 163-172. Comité des Travaux historiques, 1914, p. CLXXIV-CLXXV et p. 571-589. 
pour éclaircir les Pontiques, II, 3, 83-84 et savoir s'il y a eu un exil d'Ovide à l'île d'Elbe ${ }^{29}$. Et à plusieurs reprises, il évoque de nouveau ses repérages ostiens, notamment quand les fouilles finalement entreprises par les archéologues italiens viennent en partie confirmer ces hypothèses : «L'avouerai-je ? je n'osais pas souhaiter de l'archéologie une vérification aussi rapide et complète ", proclame-t-il au sujet de l'emplacement de la colonie primitive d'Ostie tel qu'il l'avait déterminé ${ }^{30}$. Bien plus tard, quand Jérôme Carcopino est à Vichy comme secrétaire d'État à l'Éducation (24 février 194118 avril 1942), il fait voter la loi du 27 septembre 1941 qui décide d'un contrôle des fouilles archéologiques à travers le territoire français, et qui crée, à cette fin, une administration spéciale. Les années romaines de Carcopino ont certainement contribué à penser une première fois la nécessité de cette loi.

En Italie, l'historien prend donc ses premières marques en archéologie. La fonction technique du voyage semble primer sur toute autre considération, en particulier esthétique : la découverte de l'Italie le fait s'exalter un peu, mais sans trop : qui veut voir Carcopino en admiration devant des paysages doit se contenter de quelques notations, dans des ouvrages " grand public " semblables au guide d'Ostie qu'il écrit en 1929. Dans ce guide et dans l'évocation des anciens docks, l'auteur se permet un développement lyrique qui lui fait dépeindre " le splendide déroulement de toutes ces ruines, dont les briques, suivant les heures, brillent doucement ou s'embrasent sous l'immense ciel de la campagne romaine [...] [et] l'étonnant mariage de la nature et du souvenir, du site et de l'histoire, des architectures écroulées et de la lumière immortelle 31 ». Après lui, d'autres « Romains » en disent plus sur les sentiments ressentis sous le ciel italien, à l'image de Jacques Heurgon ${ }^{32}$. L'historien se démarque de même des diplomates qui

29 «Malheureusement, je l'avoue, toutes mes tentatives de découvrir, soit sur la Via Appia, soit sur ce qui deviendra la Via traiana, une localité dont la dénomination se rapproche d'une forme verbale d'où celle d'Aethalis Ilva a pu procéder, sont demeurées vaines ", in J. Carcopino, Les Bonnes Leçons, Paris, Les Belles Lettres, 1990, 2e éd., p. 32 (1 re éd. 1968).

30 "A la fin de l'année 1922, M. Calza a exhumé autour du forum, au centre de l’Ostie des Césars, les substructions de la colonie primitive. L’emplacement que je lui avais hypothétiquement assigné avant la fouille est maintenant vérifié par la fouille. Sa forme est celle de la colonie militaire, de la base navale que j'avais prédite en 1911 " (in Ostie. Les Visites d'art, p. 27).

31 Carcopino, Ostie. Les Visites d'art, p. 22.

32 Sa correspondance avec Jean Tardieu est à cet égard révélatrice. Se reporter à la lettre du 23 octobre 1928 ( "Rome me leurre surtout de l'attrait d'une vie silencieuse et docte, mais animée au-dedans d'une flamme sincère, laissant peu de livres derrière soi, mais graves. ») et à celle du 25 octobre 1933 (" Je ne sais pas si tu peux imaginer ce qu'est [Rome] pour moi, où j'ai passé deux années de découverte et de plénitude, deux années où je ne sais pas si je dois dire que je me suis perdu ou trouvé (c'est peut-être la même chose), puisque c'est alors que je me suis détaché de moi-même pour rejoindre, enfin, une réalité qui me dépassait [...] Rome est pour moi d'une certitude infinie. " (in J. TARDIEU - J. HEURGON, Le ciel a eu le temps de changer. Correspondance 1922-1944, Paris, Mimec, 2004, p. 114 et 
sont ses contemporains et qui s'extasient sur Rome; Wladimir d'Ormesson est un de ceux-là, pour qui « l'amour qu'on porte à Rome a quelque chose de physique 33 ".

Nulle part Carcopino ne s'étend sur l'effet que produit sur lui la contemplation des ruines. Les ruines ne lui donnent pas la fièvre ${ }^{34}$. Au contraire, l'historien se sent à son aise dans Rome, cette Rome qui est surtout, à ses yeux, celle des instituts étrangers et des compétitions entre antiquisants.

\section{Rome dans une carrière}

À Rome, la carrière de Jérôme Carcopino prend de l'ampleur ${ }^{35}$. Trois ans après la soutenance de sa thèse, il est directeur intérimaire du Farnèse pour une courte période (1922-1923). Puis il devient professeur à la Sorbonne et membre de l'Académie des Inscriptions et Belles Lettres (1930). Enfin, en 1937, il prend la succession d'Émile Mâle à la tête de l'École française. La direction de Mâle 36 a duré presque quinze ans (1923-1937), puis l'historien de l'art et de Rome et ses vieilles églises 37 a été atteint par la limite d'âge (75 ans). Avant Mâle, Mgr Duchesne est directeur 38 de 1895 à 1922. À cause de la guerre, la direction de Carcopino est bien plus brève. Ceci dit, des éléments de continuité entre ces trois directeurs sont facilement repérables : le fait

p. 173). Après Heurgon, l'enthousiasme de la rencontre avec des lieux chargés de souvenirs antiques, comme Nole, se lit chez P. FABRE ( c'est, écrit ce dernier, dans ce paysage lumineux et paisible, dans cette plaine fertile que domine l'exaltante harmonie des montagnes, auprès de ces monuments dont le plan s'inscrit encore sur le sol, [que Paulin] a médité, qu'il a écrit, qu'il a prié, qu'il est mort. ", in Saint Paulin de Nole et l'amitié chrétienne, Paris, De Boccard, 1949, p. 1), ou chez J. BÉRARD, qui décrit avec enthousiasme les temples de Sélinonte, in La colonisation grecque de l'Italie méridionale et de la Sicile dans l'Antiquité : l'histoire et la légende, Paris, De Boccard, 1941, p. 264.

W. D’Ormesson, De Saint-Pétersbourg à Rome, Paris, Plon, 1969, p. 303.

Cf. l'article de J.-P. Clément, supra, p. 179-189.

Jérôme Carcopino construit soigneusement sa carrière tout au long de sa vie. Directeur de l'École de Rome, il est aussi membre résidant, puis membre honoraire de la Société Nationale des Antiquaires de France, membre de l'Institut de France, membre de l'Académie française, membre ordinaire de l'Institut archéologique allemand, membre associé de l'Académie Royale de Belgique, membre d'honneur de l'Académie Pontificale d'archéologie, Docteur Honoris Causa de l'Université d'Oxford, membre associé de l'Académie dei Lincei et de l'Académie des sciences de Turin, correspondant de l'Academia de la Historia de la République argentine. Voir Émile Mâle (1862-1954). La construction de l'euvre, Rome et l'Italie, Paris, De Boccard, 2005, 360 p. l'EFR, Rome, De Boccard, 1992, 757 p. 
d'avoir été membre de l'EFR, les convictions chrétiennes et l'attention portée aux élèves ${ }^{39}$. Les trois se succèdent de surcroît à l'Académie française.

Quand Jérôme Carcopino vient assumer sa charge de directeur, le régime fasciste se durcit et les relations franco-italiennes sont tendues, ce que décrit bien l'ambassadeur François-Poncet, racontant par exemple qu'il a aperçu [...], « un cercueil recouvert du drapeau français sous les fenêtres du Farnèse 40 ". Au même moment des étudiants de Turin distribuent des brochures anti-françaises ${ }^{41}$. Mussolini interdit qu'à Naples soit mis en scène L'Aiglon. Des touristes français, qui lisent innocemment l'Osservatore romano, sont interpellés par la police ${ }^{42}$. Cette francophobie s'explique de plusieurs manières : d'une part, la France n'a pas unanimement approuvé la conquête de l'Éthiopie et, d'autre part, l'Italie revendique la Tunisie, la Corse et Djibouti dans plusieurs campagnes menées à partir de l'automne 1938.

Malgré tout, les mondanités auxquelles doit se plier tout représentant de la France à l'étranger ne cessent pas : Carcopino fréquente les salons romains, reçoit les félicitations du Comte Ciano à propos « du retentissement de [son] César en Italie 43 ». Il va visiter l'éminent romaniste et sénateur à vie Ettore Pais, et boit du vin à la grecque, avec un cratère, en compagnie de Ludwig Curtius 44.

Son action, quoique courte, dans l'histoire de l'École est marquante : il met de l'ordre dans la bibliothèque et crée un poste de bibliothécaire, il fait venir - c'est le premier - un membre étranger ${ }^{45}$, imagine un recrutement plus large, inspire des sujets ${ }^{46}$, œuvre pour une plus grande ouverture à l'archéologie africaine, organise des

39 Les élèves antiquisants de l'École dirigée par Carcopino sont Jean Bérard (Sicile et GrandeGrèce), Raymond Bloch (Bolsena), Marie Collon Janin, devenue Mme Jean Bérard (qui étudie la colonie juive de Rome, mais qui ne va pas jusqu'à la thèse), Pierre Grenade (les origines du principat), Pierre Grimal (les jardins romains), Julien Guey (le Septizonium), Georges Lopuszanski (Bas-Empire, mais ne produit ni mémoire ni thèse), Jules Paoli (droit romain), Gilbert Picard (les trophées romains et Mactar), Robert Schilling (Vénus). A. François-Poncet, Au Palais Farnèse. Souvenirs d'une ambassade à Rome. 1938-1940, Paris, Fayard, 1961, p. 47.

41 Ibid., p. 110.

42 Ibid., p. 160-161.

43 J. CarCOPINO, Souvenirs de sept ans (1937-1944), Paris, Flammarion, 1953, p. 31.

44 Carcopino participe aux manifestations du bimillénaire d'Auguste depuis la France. En juillet 1937, il est entre Monaco, La Turbie, Arles et Vienne pour ces commémorations (cf. Comptes rendus de l'Académie des Inscriptions et Belles Lettres, 1937, p. 186-187).

46 Avant de diriger l'École française, Carcopino donnait déjà aux futurs élèves des thèmes de recherche (c'est le cas de l'enquête sur Capoue proposée à Jacques Heurgon). Jean Gagé (membre 1925-1928) lui doit aussi son sujet sur Apollon (Apollon romain. Essai sur le culte d'Apollon et le développement du "ritus graecus " à Rome, des origines à Auguste, Paris, De Boccard, 1955, 741 p.). 
cycles de conférence ${ }^{47}$. Enfin, en 1940, quand la guerre rend impossible toute présence française dans la capitale italienne, c'est lui qui refuse la délocalisation de l'École en Algérie ou à Aix-en-Provence.

Quand il devient le directeur du Farnèse, la motivation de Carcopino n'est pas uniforme : le poste officiel et prestigieux l'attire à coup sûr, mais il souhaite en outre s'occuper du rayonnement culturel de la France à l'étranger, d'autant que le directeur de l'École de Rome exerce une autorité morale sur le Lycée français Chateaubriand, et sur les Instituts français de Florence et de Naples. Année après année, l'Académie des Inscriptions exprime, dans ses Comptes rendus, le même souci de maintenir l'éclat scientifique de la France hors de ses frontières, et donne des nouvelles d'une École presque toujours appelée " notre " École de Rome, ou " notre laborieuse colonie du Palais Farnèse 48 ".

Depuis quelques années, l'historien a précisé sa pensée politique - conservatrice en fréquentant les déjeuners Hervieu, où il a fait la rencontre du Maréchal Pétain, mais aussi en publiant dans la Revue des Deux Mondes, en s'intéressant au cercle Fustel de Coulanges constitué de proches de l'Action française ${ }^{49}$, en signant la pétition demandant au gouvernement français le retrait des sanctions infligées à l'Italie à la suite de la guerre d'Éthiopie ${ }^{50}$. Ses années de direction romaine ne font pas naître, chez lui, une nouvelle prise de conscience politique. Ici, l'attitude du directeur differe de celle de certains élèves de l'EFR dans les années 1930. Dès 1933, Jacques Heurgon, dans une lettre, rapporte en effet des propos sur le nazisme naissant tenus par quelques Juifs allemands dont il a croisé le chemin à Rome ${ }^{51}$. De l'Italie fasciste, Henri-Irénée Marrou envoie, quant à lui, sous le pseudonyme de François Queylard, des témoignages, que les revues Politique et Esprit publient ${ }^{52}$. Membre de l'École française sous la direction de Carcopino, Raymond Thouvenot, qui s'est attelé à étudier la Bétique antique, dédie

47 Cf. Comptes rendus de l'Académie des Inscriptions et Belles Lettres, 1940, p. 259. Parmi les conférenciers : Henri Bordeaux, Émile Mâle, Abel Bonnard (qui, à Vichy, succède à Carcopino à l'Éducation nationale).

48 Cf. Comptes rendus de l'Académie des Inscriptions et Belles Lettres, 1937, p. 207.

49 Pour connaître les habitués des déjeuners Hervieu, cf. CARCOPINO, Souvenirs de Sept ans. 1937-1944, p. 158 et s. Dramaturge, Paul Hervieu est académicien en 1916. La personnalité politique de Carcopino, ses liens avec le cercle Fustel sont analysés par S. CORCYDebray, Jérôme Carcopino, un historien à Vichy, Paris, L'Harmattan, 2001, 550 p. Sur le Cercle lui-même et ses liens avec l'Antiquité, cf. C. VALENTI, "L'Action française et le Cercle Fustel de Coulanges à l'école de l'Antiquité (première moitié du XXe siècle) ”, Anabases 4 (2006), p. 49-64. Cf. Carcopino, Souvenirs de Sept ans, p. 22.

Lettre à Jean Tardieu, 19 novembre 1933, in TARDIEU - HEURGON, Le ciel a eu le temps de changer, p. 174.

Les articles que Marrou envoie à Esprit portent sur " L'Italie prolétaire et fasciste " (décembre 1935) et "Le fascisme italien et la femme" (juin 1936). Cf. P. Riché, HenriIrénée Marrou, Un historien engagé, Paris, Le Cerf, 2003, p. 50 et p. 54. 
sa thèse aux "Andalous de toutes les classes 53 " et traduit sa hantise du franquisme au détour d'un compte rendu 54 . Pendant la guerre même, Robert Schilling, autre élève de Carcopino, glisse une attaque anti-allemande et anti-Quellenforschung dans une édition du poème la Veillée de Vénus 55 .

On imagine bien que Jérôme Carcopino, à une place aussi en vue, n’a pu bénéficier d'une grande liberté de ton. Mais s'il avait pu s'exprimer sans contrainte, son conservatisme politique l'aurait sans doute freiné. Même s'il n'a jamais manifesté de claire opinion politique de 1937 à 1940, on peut penser que sa Vie quotidienne, seul ouvrage écrit intégralement depuis le palais Farnèse, rend compte d'un esprit de catastrophe imminente, à l'approche de la guerre. Cette tristesse, cette inquiétude, Marrou les a ressenties aussi, au point de faire de l'étude de la décadence romaine une exigence, après avoir décrit en ces termes l'avant-guerre dans les Fondements d'une culture chrétienne, "Nous vivions, se souvient-il, dans une atmosphère d'Apocalypse ; on entendait de toutes parts des craquements qui semblaient annoncer l'écroulement du monde 56. "À la lecture de Marrou, d'autres savants ont renchéri sur le thème de la dégénérescence présente : ainsi Pierre de Labriolle redoute-t-il pour son époque et sa culture "quelque retour des "Dark Ages" 57 ".

R. Thouvenot, Essai sur la province romaine de Bétique, Paris, De Boccard, 1940, p. 3. Compte rendu qui lui permet de se poser quelques graves questions : « Des érudits espagnols que nous avions coutume de rencontrer dans [les] vastes salles [du Musée archéologique de Madrid] si accueillantes au travailleur comme au touriste, combien survivent maintenant? Et que reste-t-il du musée lui-même. » (R. Thouvenot, "Compte rendu de Pierre Paris, Le Musée archéologique national de Madrid, Paris, Les éditions d'Art et d'Histoire, 1936 ”, Comptes rendus de l'Académie des Inscriptions et Belles Lettres, 1938, p. 133).

"Pierre Pithou [qui découvre le manuscrit du poème au XVI siècle] s'était bien adressé aux médecins [...] en faveur du poème malade. Je doute qu'il eût appelé à son chevet les “chirurgiens ” qui depuis le XIXe siècle ont disséqué à l'envi le poème. À une lacune près, le texte transmis présente un ensemble naturel. Nous avons donc renoncé à tout essai arbitraire de "reconstitution " et nous offrons au lecteur le Pervigilium dans l'ordre originel. Loin d'adapter une solution de paresse, nous croyons servir le poème avec le respect loyal, fide, que réclamait en 1577 son premier éditeur, le français Pierre Pithou. " (in "Avantpropos ", écrit en septembre 1943, de La Veillée de Vénus, texte établi, traduit et commenté par Robert Schilling, Paris, Les Belles Lettres, 1961, p. XIV). H.-I. Marrou, Fondements d'une culture chrétienne, Paris, Blond \& Gay, 1934, p. 13.

Pierre de Labriolle écrit : «La seule réflexion un peu mélancolique que [cette thèse] suggère, la voici : M. Marrou a eu l'occasion de dresser, en divers endroits, de son travail le tableau clinique d'une décadence, considérée exclusivement du point de vue intellectuel. Un symptôme qu'il relève souvent, c'est l'abus du manuel, des épitomés, des compilations [...] mais qu'apprend donc aujourd'hui notre jeunesse, dans les intervalles de ses "loisirs", sinon des mémentos, des paragraphes, des analyses d'auteurs qu'elle effleure à peine [...] Faut-il conclure de ces analogies que la culture latine du IVe siècle finissant n'était pas tellement absurde ? Ou bien devons-nous nous demander si la nôtre a bien le droit de s'enorgueillir de ses méthodes, et si elle ne prépare pas quelque retour des Dark Ages. " " Compte rendu d'Henri-Irénée Marrou, Saint Augustin et la fin de la culture antique, Paris, De Boccard, 1938, 620 p. ", Comptes rendus de l'Académie des Inscriptions et Belles-Lettres, 1938, p. 154). 
Dans une Rome fort différente de celle qu'il a connue comme farnésien, le directeur Carcopino poursuit donc sa brillante carrière et écrit un livre - La vie quotidienne à l'apogée de l'Empire - qui s'inscrit dans un contexte particulier de crises.

\section{Rome antique : « quotidienne » et condamnée}

Cette Vie quotidienne est une première, jaillie de l'idée géniale d'un directeur éditorial : Maurice Labouret ${ }^{58}$. Trois auteurs donnent à cette collection des Vies quotidiennes son coup d'envoi : Abel Lefranc pour la Renaissance 59, Jean Robiquet pour la Révolution ${ }^{60}$, Jérôme Carcopino pour Rome. Des trois, le romaniste est celui qui remporte le plus de succès à travers le monde et celui qui est encore réédité, alors qu'il n'y a plus d'autre tirage de la Vie quotidienne d'Abel Lefranc après 1963, après 1964 pour la Vie de Jean Robiquet. Cette collection est lancée quand l'histoire économique et sociale fait ses premiers pas, et dans une période de vogue de la vulgarisation scientifique. Quelques années auparavant, il était davantage question de "vie privée ", décrite dans des livres parfois romancés ou dans des manuels et des dictionnaires qui font la part belle aux realia.

Lorsque paraissent les premières Vies quotidiennes, de nombreux scientifiques écrivent des livres pour un large public, à mi-chemin entre la science et la philosophie ${ }^{61}$. Et la collection "Que sais-je ?" est lancée par les Presses Universitaires de France en 1941. Les Farnésiens, de leur côté, ont pris part à ce mouvement et n’ont pas ménagé leurs efforts en matière de vulgarisation du savoir : Carcopino lui-même rédige des guides de voyage 62. Un peu plus tard, Pierre Grimal a écrit plusieurs Que sais-je ? ${ }^{63}$, des romans historiques et un livre de voyage intitulé Partons pour Rome ${ }^{64}$; Raymond Bloch un opuscule sur Le Mystère étrusque ${ }^{65}$; Jacques Heurgon 66, Robert Etienne ${ }^{67}$,

58 Cf. J. Mistlen, La librairie Hachette de 1826 à nos jours, Paris, Hachette, 1964, rééd. 1979, p. 354.

59 A. LeFrAnC, La vie quotidienne au temps de la Renaissance, Paris, Hachette, 1938, 253 p.

60 J. RobIQUeT, La vie quotidienne au temps de la Révolution, Paris, Hachette, 1939, 256 p.

61 Le géologue chrétien Pierre Termier remporte ainsi, en 1929, un immense succès avec La Vocation de savant (Paris, Desclée de Brouwer, 1925, 263 p.). André Georges, Louis de Broglie, Rémy Collin, Alexis Carrel illustrent le genre eux aussi.

62 Cf. supra, n. 22.

63 Dans la série des "Que sais-je ? ", P. GRIMAL a donné plusieurs titres : La Vie à Rome dans l'Antiquité (1953), L'art des jardins (1954), Les Villes romaines (1954), Le siècle d'Auguste (1955), La littérature latine (1965) et Le théâtre antique (1978).

P. GRIMAL, Nous partons pour Rome, Paris, PUF, 1962, 239 p.

R. BLOCH, Le mystère étrusque, Paris, Le club français du livre, 1956, 246 p.

Heurgon, La vie quotidienne chez les Étrusques. L'auteur dédie son livre à Jérôme Carcopino.

R. ÉTIEnNe, La vie quotidienne à Pompéi, Paris, Hachette, 1966, 491 p. 
Gilbert Picard 68 ont également participé à l'enrichissement de la collection des Vies quotidiennes d'Hachette. Signe supplémentaire d'un intérêt pour la vulgarisation : les anciens "Romains » ont enregistré des émissions radiophoniques 69.

Pour rendre aisée et vivante la lecture de son livre, Carcopino, qui s'est limité au début du II siècle après J.-C., a largement pioché dans Juvénal et dans Martial. Les descriptions pittoresques, les anecdotes savoureuses étaient toutes là, prêtes à l'emploi. L'auteur utilise le Juvénal de la première Satire pour le portrait méprisant des clients empressés de bon matin, parle de " tourbillons de salamalecs 70 » et exploite tous les recoins de la sixième Satire, celle qui prend comme objet les femmes, leurs ridicules et leurs perversions. En bien des pages de l'ouvrage, l'homme romain paraît " un ", toujours le même, et toujours victime face à une nuée nocive de femmes corrompues : " elles ne tardaient pas à manquer à la foi qu'elles auraient dû lui promettre [nous soulignons] 71 ", déplore ainsi Carcopino. Cette déchéance s'expliquerait par le fait que le " soin des enfants, cette sauvegarde de la femme, échappait à la matrone dès leur sortie du bas âge 72 ". Afin d'alléger son réquisitoire contre l'" espèce " féminine romaine, l'historien dresse une liste des femmes romaines dignes d'imitation : Sabine, Pauline qui suit Sénèque dans la mort, Arria l'Aînée, la Bretonne Claudia Rufina, Calpurnia qui est la femme de Pline le Jeune ${ }^{73}$.

68 G. et C. Charles-PiCARD, La vie quotidienne à Carthage au temps d'Hannibal. II siècle avant J.-C., Paris, Hachette, 1958, 271 p.

69 Carcopino traite le sujet "Les Romains et nous " dans une série d'émissions de " radiophonie scolaire ", enregistrées en 1937, tandis qu'Albert Grenier parle de "Rome et les Gaulois" et de "Paris gallo-romain ”. Cf. Revue des études latines, 1937, p. 59 sq. CARCopino, La Vie quotidienne à Rome à l'apogée de l'Empire, p. 206.

Ibid., p. 113.

Ibid., p. 125.

73 Plus tard dans son ouvre, l'historien tient de nouveau à faire l'éloge de quelques hautes figures féminines, comme dans ses Promenades historiques aux pays de la Dame de Vix (Paris, L'artisan du livre, 1957, 114 p.) : "À Avaricum, en 52 av. J.-C., ce sont les femmes qui ont empêché l'évacuation de la ville. À Gergovie, ce sont elles qui ont jeté robes et bijoux aux pieds de l'assaillant romain pour en retarder l'élan, puis qui, du haut des remparts, ont encouragé la victoire de leurs hommes. Encore au IVe siècle, Ammien consacrera la réputation des guerrières qu'elles s'étaient acquises en se jetant, quand il le fallait, dans la mêlée et en frappant l'ennemi d'un poing aussi redoutable que l'engin de catapulte lâché par sa corde. Tout le monde se souvient de la vaillance d'Éponime qui, au pays de Langres, concourut de sa personne à la révolte suscitée contre Rome par son mari, Julius Sabinus, et, après la défaite de celui-ci, en partagea, neuf ans de suite, en des cachettes sinistres, la misérable existence de hors-la-loi, en attendant de marcher, tête haute, à côté de lui, au supplice ordonné par Vespasien ; et, si l'origine en est moins connue, il n'est pas moins admirable l'héroïsme de l'Arlésienne Pauline qui refusa la grâce impériale et voulut suivre dans la mort Sénèque, son mari, condamné par Néron» (p. 92). 
En plus de quelques «belles » figures, Carcopino fait preuve d'indulgence à l'égard des grands monuments : le forum de Trajan est " une de ces cimes de l'art où se rencontrent les constructeurs des meilleures époques 74 ", la cloaca maxima, " un puissant chefd'œuvre auquel collaborèrent, avec la longue expérience amassée par les Étrusques dans le drainage de leur maremme, la hardiesse et la patience du peuple romain ${ }^{75}$ ", et les thermes :

je suis persuadé, affirme l'auteur, que les thermes impériaux ont apporté aux masses un puissant bienfait. Dans leur majesté éclatante de marbre, ils n’ont pas été seulement le splendide palais de l'eau romaine. Ils furent surtout le palais du Peuple [...] où, jadis, les Romains, acquérant tout ensemble le goût de la netteté physique, des sports utiles et de la culture désintéressée, ont pu, pendant plusieurs générations, retarder leur décadence par un retour au vieil idéal qui avait inspiré leur grandeur dans le passé, et que, dans le présent, leur propose toujours Juvénal d'un "esprit sain dans un corps sain" 76.

Sauvée par quelques rares Romains et par les beaux monuments, la Ville a tout de même ceci de positif qu'elle échappe, selon Carcopino, aux conflits sociaux : "un souffle égalitaire [...] avait toujours rapproché les plus nobles demeures et les maisons les plus vulgaires 77 ». Ce constat est dressé sans que soient abordées les questions économiques en terme d'histoire sociale, ce qu'André Piganiol, attaché à l'école des Annales, ne manque pas de souligner. Piganiol écrit ainsi qu' "il n'eût pas été sans intérêt de compléter cette étude [Vie quotidienne] par une étude du prix de la vie " et s'interroge : "Aurait-on pu aller jusqu'à esquisser un budget moyen [de l'homme romain] 78 ?"

La Vie quotidienne à Rome a donc le moralisme des manuels de la fin du XIXe siècle, du type du Manuel des antiquités romaines de Joaquim Marquardt, qui dépeint une société qui se craquelle, avec des Romains qui, dès l'époque de Cicéron, ne seraient que des "viveurs esclaves de leur bouche ", formant des " générations au visage blême, aux joues flasques, aux yeux tuméfiés 79 ». Mais là où Marquardt rejette en bloc ce que sont devenus les Romains au tournant de notre ère, Carcopino distribue bons et mauvais points.

74 CARCOPINO, La vie quotidienne à Rome à l'apogée de l'Empire, p. 11.

75 Ibid., p. 57.

76 Ibid., p. 304.

77 Ibid., p. 43.

78 Cf. Revue historique (1939, p. 286). L'absence d'intérêt pour ce domaine historique n’a pas toujours été une donnée des travaux de Jérôme Carcopino. Au début de sa carrière, Carcopino s'est occupé d'histoire économique dans La loi de Hiéron et les Romains (Paris, De Boccard, 1914, 307 p.) et dans une étude d'histoire moderne (Une terre normande à la veille de la révolution, Paris, A. Aubert, 1906, 55 p.).

79 J. Marquardt, La Vie privée des Romains, Paris, E. Thorin, traduit de l'allemand par Victor Henry, 1892 ( $1^{\text {re }}$ éd. 1879), 2 vol., p. 82 [tomes XIV et XV du Manuel des antiquités romaines dirigé par Theodor Mommsen]. 
À la fin, le malaise de l'historien face au Romain quotidien est tout de même si palpable qu'il sort métaphoriquement de Rome, pour se réfugier à Lanuvium. Ainsi, dans les dernières pages de sa Vie quotidienne, Jérôme Carcopino passe sans transition du récit du dîner orgiaque de Trimalcion raconté par Pétrone à l'éloge des agapes selon Tertullien, avant d'achever son propos par l'évocation des repas du collège funéraire de Lanuvium, formé en 133, et dont nous connaissons les statuts. Carcopino écrit alors :

Les vertus de l'ancienne Rome ont l'air de se ranimer dans cette association de pauvres gens de la banlieue de Rome au temps d'Hadrien : la sobriété, la discipline, l'urbanité. Il semble même y voir éclore un sentiment nouveau, tout à l'honneur des " collègues " de Lanuvium : celui de la fraternité qui les unit dans la vie comme il devra, plus tard, les réunir dans la mort, en prévision de laquelle ils se sont rassemblés pour subvenir ensemble aux frais de leurs funérailles respectives et mériter en commun la récompense du salut de l'au-delà 80 .

Toute la Vie quotidienne de Carcopino est écrite sous l'influence d'une source très conservatrice, Juvénal. Quand l'écrivain ancien affirme, dans sa deuxième Satire, qu'il lui " prend envie de [s']enfuir d'ici par delà les Sarmates et l'Océan glacial 81 ", tant Rome lui est intolérable, il est facile d'imaginer dans un même esprit l'auteur moderne tenté par une échappée vers le christianisme en passant par Lanuvium.

Pour Carcopino, la Rome du début du IIe siècle, c'est :

le grouillement de ses foules aux pieds de la majesté impériale, sa fièvre de l'argent, l'étalage du luxe qui recouvre ses misères, la prodigalité des spectacles où s'étire sa paresse et s'attisent ses mauvais instincts, l'inanité des divertissements intellectuels où les uns s'anémient, la frénésie de jouissances charnelles qui abêtit les autres. Mais il ne faut pas que cet éclat trompeur, ni ces ombres sinistres cachent la petite lueur qui, si faible et tremblante qu'elle fût, s'était levée parmi des âmes d'élite comme l'aube naissante d'un autre monde 82 .

C'est que, dans l'esprit de Carcopino, la vie quotidienne n'est qu'un arrêt de l'histoire, une stagnation. Il n'est pas le seul à penser en ce sens le quotidien. Ainsi, un autre Farnésien, Eugène Albertini, écrit-il, toujours en 1939 et au sujet de Bénévent, que « ce

$80 \quad$ Ibid., p. 324.

81 Juvénal, Satires, Paris, Les Belles Lettres, Texte établi et traduit par Pierre de Labriolle et François Villeneuve, 1994 (13e tirage), $1^{\text {re }}$ éd. 1921, p. 14.

82 Carcopino, La Vie quotidienne à Rome à l'apogée de l'Empire, p. 166-167. De telles descriptions ont pu inspirer Federico Fellini dans son travail sur le Satiricon de Pétrone. Élaborant son film, Fellini a dit qu'il devait beaucoup à la Vie quotidienne de Carcopino. Le thème de la déliquescence morale réapparaît dans le film du réalisateur italien. Cf. Grimal, Ourliac, CarCopino, Jérôme Carcopino, un historien au service de l'humanisme, p. 83. 
que les documents archéologiques nous permettent d'atteindre, c'est la Bénévent impériale, du II et du III siècle, qui a une vie quotidienne mais qui n'a plus d'histoire 83 ».

D'une certaine façon, Rome, telle qu'elle est décrite dans la Vie quotidienne, est en contraste avec la Rome de la Mostra Augustea della romanità, organisée par le régime fasciste pour les deux mille ans d'Auguste ${ }^{84}$. Dans la Mostra montée au Museo Nazionale Romano, la vie romaine de tous les jours n'est pas peinte des mêmes couleurs. Les demeures sont raffinées, une maison de Pompéi est même reconstruite. La vie familiale est admirable. L'Urbs est un prodige d'urbanisme, qu'une grande maquette permet de surplomber. Selon le message transmis, Rome, toute son histoire durant, n'a connu ni déclin ni catastrophe, qu'elle soit lente ou brutale (aucun corps pompéien n'est exposé). $\mathrm{Et}$, en toute logique, les premiers lecteurs italiens de cette Vie quotidienne s'offensent de la noirceur du tableau de la Ville que donne l'historien ${ }^{85}$. Finalement, la Vie quotidienne de Carcopino parait aujourd'hui très datée sous bien des aspects : par son extrême moralisme, par son ton de censeur qui classe les vices et les vertus des Romains ${ }^{86}$, par ses allusions entendues à l'attention de lecteurs pourvues d'une solide culture classique ${ }^{87}$.

La condamnation des mœurs romaines de l'époque impériale devient par la suite un motif récurrent des manuels d'histoire écrits sous Vichy, lorsque Carcopino est ministre de l'Éducation, deux ans après la publication de la Vie quotidienne. Ainsi, le manuel Hatier de 5e édité en 1942 dit bien que sous l'empire : "Une vie luxueuse, amollie par les plaisirs, s'était substituée à l'existence simple et rustique des anciens Romains. La fréquence des divorces et l'inconduite des époux ruinaient le prestige de la famille. On ne croyait plus aux dieux ou l'on faisait semblant d'y croire ${ }^{88}$. "Selon le manuel Hachette publié la même année, dès la fin de la République, « la désorganisation de la famille et les progrès de l'irréligion alarment les admirateurs des vertus traditionnelles 89 \%. Au chapitre de la "Transformation des mœurs à la suite des conquêtes ", le manuel Masson fait entendre les mêmes accents, stigmatisant chez les

83 Voir E. Albertini, " Rapport sur les travaux de l'École française de Rome, en 19371938 ", in Comptes rendus de l'Académie des Inscriptions et Belles Lettres, 1939, p. 252. Eugène Albertini évoque ici l'activité de Michel Labrousse.

Cf. Mostra augustea della romanità : catalogo, Roma, Colombo, 1938, 770 p.

G. CALZA, chargé du compte rendu de l'ouvrage dans le Bolletino della Commissione archeologica del governatorio di Roma (1940, p. 135-136), fait par exemple remarquer que «Soltanto cento anni fa, le condizioni di netteza, di decenza, di elementare conforto in tutte le capitali europee fossero inferiori a quella di Roma imperiale» (p. 136).

Voir Chapitre II "Le Mariage, la femme et la famille : vertus et vices" de CARCOPINO, $L a$ Vie quotidienne à Rome à l'apogée de l'Empire, p. 97-124.

Un exemple : "Tout le monde, dit l'historien, connaît par cœur la tirade fulminée par Juvénal contre "la tourbe dégénérée des enfants de Rémus" ", in CARCOPINO, La Vie quotidienne à Rome à l'apogée de l'Empire, p. 238.

L. Harmand et A. Bossuat, Rome et les débuts du Moyen Âge. Classe de 5 ìme, Paris, Hatier, 1942, p. 116.

A. AlBA, Rome et les débuts du Moyen Âge. Classe de 5ème, Paris, Hachette, 1942, p. 132. 
Romains « le désir de jouir, si longtemps contenu, [qui] éclata dans les appétits primordiaux et animaux : la gloutonnerie, la vanité, le besoin d'émotions violentes, cette ostentation des choses coûteuses et cette profusion de la richesse faite uniquement pour montrer qu'on la possède, le luxe absurde et grossier des parvenus 90 ".

Tout bien considéré, ce ne sont pas les villes, ces vagues ensembles humains, qui comptent dans l'historiographie carcopinienne, mais les grands hommes. Carcopino n'a-t-il pas confié avoir choisi l'histoire antique " parce que l'enquêteur [...] n'y est pas à la merci d'une liasse d'archives inaccessibles ou d'une correspondance privée que ses propriétaires se refusent à communiquer 91 " ? Ces grands hommes sont parfois accompagnés de femmes illustres, et les portraits que l'historien fait de ces acteurs "véritables " de l'histoire sont souvent nuancés 92 . Dès son étude sur l'ostracisme, Clisthène était admiré parce que rattaché à cette poignée d'hommes qui, aux yeux de l'antiquisant, font l'histoire ${ }^{93}$. Ces hommes sans qui il n'y aurait pas d'histoire selon Carcopino ne sont pas nécessairement romains : le rôle joué par quelques Grecs, dont Clisthène, ou Pyrrhus ${ }^{94}$, est salué. Mais Hannibal 95, Massinissa 96, Genséric 97 ont droit aussi à la considération de l'historien...

Ce n'est pas le répétitif de chaque jour qui intéresse Carcopino, mais les moments forts, les grandes dates. Il n'y pas, pour lui, de mystère à élucider dans la grisaille du jour le jour. Jérôme Carcopino veut bouleverser 98 l'histoire romaine, et les rues mal entretenues, l'animation du cirque, les tavernes de Rome ne se prêtent pas à de nouvelles interprétations sensationnelles. À mots couverts, il s'en prend souvent à l'his-

90 P. Hallynck et M. Brunet, L'Antiquité. II. Rome-Haut Moyen Âge. Classes de 5 e classique et moderne, Paris, Masson, 1942, p. 89.

91 Cité par CorCY-Debray, Jérôme Carcopino. Un historien à Vichy, p. 20.

92 La bibliographie de Jérôme Carcopino comporte plus de mille titres. Sur ce millier de titres, seulement une trentaine de références portent sur la ville de Rome proprement dite. Voir bibliographie exhaustive dans Hommage à la mémoire de Jérôme Carcopino, Paris, Les Belles Lettres, 1977, p. 349-411.

93 Clisthène « était, comme Solon, une de ces intelligences heureuses, faites de pondération enthousiaste et de hardiesse sereine, qui préparent l'avenir sans renier le passé, et dans le présent où ils vivent, font des révolutions énormes avec des timidités de réactionnaires ", cf. "Histoire de l'ostracisme athénien " in Mélanges d'histoire ancienne, Paris, Felix Alcan, Université de Paris, Bibliothèque de la Faculté de Lettres, t. XXV, 1909, p. 83-267. Voir l'étude "Pyrrhus, conquérant ou aventurier ?" (p. 9-108) in J. CARCopino, Profils de conquérants, Paris, Flammarion, 1961, 410 p.

95 Cf. "Grandeur et faiblesses d'Hannibal ”, ibid., p. 109-238.

96 “Massinissa, l'allié des Romains, dont le génie continue l'œuvre de Carthage et anticipe sur les méthodes de l'Islam " (CARCOPINO, Les Bonnes Leçons, p. 60).

97 Cf. "Genséric et les Vandales conquérants de l'Afrique", in CARCOPINO, Profils de conquérants, p. 357-409.

98 De la quatrième étude de Passion et politique chez les Césars, sur "Le bâtard d'Hadrien et l'hérédité dynastique chez les Antonins", Carcopino dit qu'elle «bouleverse la conception qui, jusqu'ici, dominait le siècle des Antonins" (p. 6). 
toire qu' inspirent les Annales de Marc Bloch et Lucien Febvre 99. De plus, Carcopino aime livrer de beaux morceaux de rhétorique, ce que ne permettent pas des sujets aussi triviaux que l'approvisionnement ou les égouts de la Ville. Il préfere se trouver dans les salons impériaux, les palais, les séances agitées du Sénat plutôt que dans la rue populeuse qu'habite Martial ${ }^{100}$.

Par conséquent Rome a pour l'historien une triple valeur. Tout d'abord, c'est la ville où il doit se rendre quand il décide de se faire romaniste et devient membre de l'École française. Et, au fil de sa carrière, c'est à Rome qu'il vient en priorité quand il séjourne en Italie. De plus, la Ville représente un bel espace d'ascension dans une carrière qui se définit et se joue entre Paris, la capitale italienne et l'Afrique du Nord. La Rome antique, enfin, est un personnage de l'œuvre de Carcopino, mais un second rôle, souvent même un simple décor. La lecture de la Vie quotidienne le dit nettement : à côté du christianisme naissant et de quelques consciences « supérieures ", ce qu'il reste d'admirable dans la Ville impériale, ce sont certaines prouesses architecturales : le monumental. Dans une Rome encore vivante, Carcopino n'aime que ce qui va en rester : les ruines à venir.

\begin{tabular}{l} 
Sarah REY \\
\hline Université de Toulouse II-Le Mirail \\
UFR d'Histoire, Arts et Archéologie \\
5, allées Antonio Machado \\
F-31058 Toulouse Cedex 9 \\
sreylauzerte@yahoo.fr
\end{tabular}

99 Cf. Passion et politique chez les Césars: "Quand [le lecteur] m'aura vu m’évertuer à fixer aux quantièmes une chronologie, à déployer les frondaisons d'un arbre généalogique particulièrement touffu, à déplacer ou modifier, après mûre réflexion, un mot dans une phrase latine dont ces menues mutations transforment le sens et la portée, il est possible qu'il soupire après le grand large des avenues désencombrées que dessine, devant ses curiosités, l'histoire qualifiée par un jargon barbare de " non événementielle" " (p. 6).

100 Cf. Martial, Épigrammes, XII, LVII. 